\title{
An In Vivo/In Vitro Evaluation of Teratogenic Action
}

\author{
ALLAN R. BEAUDOIN AND D. LOWELL FISHER \\ Department of Anatomy, University of Michigan, Ann Arbor, Michigan 48109
}

\begin{abstract}
Several compounds were administered to pregnant Wistar-derived rats either 24 or four hours prior to the recovery of day 10 embryos for in vitro culture in Waymouth's medium and fetal calf serum. The compounds tested were 2 -amino-1,3,4,-thiadiazole (thiadiazole), cadmium sulfate, 1,2-dibromo-2,2dichloroethyl dimethyl phosphate (dibrom), 2-(sec-Butyl)-4,6-dinitrophenol (dinoseb), lead nitrate, polybrominated biphenyls (PBB), sodium arsenate, and trypan blue. After 24 hours in culture, two thirds of the embryos were recovered for examination. The remaining one third were continued in culture until 42 hours. Recovered embryos were examined for rotation of the embryonic axis, heart rate, establishment of the visceral yolk sac circulation, somite number, growth of the limb buds, closure of the neural tube, and development of the allantois and amnion. All tested compounds inhibited the rate of development in vitro.
\end{abstract}

A variety of in vitro screening tests are currently being investigated as rapid methods to evaluate compounds for teratogenicity. One criticism raised is that in in vitro testing the dynamic relationship between the mother and embryo is lost, and modulation of the motherplacenta-embryo unit cannot take place. The procedure to be described in this paper attempts to circumvent this criticism by combining in vivo treatment with in vitro evaluation of development. The procedure allows the maintenance of the mother-placenta-embryo relationship during the time the teratogen is suspected to act.

Attempts to culture the postimplantation mammalian embryo are not new (Jolly and Lieure, '38; Nicholas and Rudnick, '38). It was not until 1964, however, that New and his coworkers (New and Stein, '64) began to publish the results of experiments that culminated in a method for the successful culture of postimplantation rodent embryos (New, '71). Continued modifications and refinements have been made in the technique for rat embryo culture until the rate of growth and differentiation in vitro are markedly similar to growth and differentiation in vivo (New et al., '76). Evaluations of the usefulness of in vitro techniques in the assessment of teratogenesis, however, have been equivocal (see reviews: Kochhar, '75, New, '78; Wilson, '78).

The present paper reports the results of experiments combining maternal teratogen treatment with in vitro embryo culture.

\section{MATERIALS AND METHODS}

Virgin female Wistar-derived rats from our colony were used. The animals were fed Tekald Rat Diet (Teklad Mills, Winfield, Iowa) ad libitum with supplemental feedings of lettuce. Water was available at all times. The day of finding sperm in the vaginal smear was designated day 0 of pregnancy. Teratogenic doses of the test compounds were administered at 10 AM on day 9 or 10 of gestation. The compounds used, their dosages, and the route of administration, are given in Table 1.

Twenty-four hours after day 9 injection, or four hours after day 10 injection, the embryos were recovered for culture following the dissection method of New and Coppola ('77). An attempt was made to culture ten embryos from each litter (except only seven from day 10 controls). Some litters, however, had fewer than ten embryos, and some embryos were damaged or lost prior to culture. Usually, therefore, fewer than ten embryos were actually placed in culture. Embryos placed in culture were dorsiflexed, had five to nine somites, a beating heart, and an open neural tube (Fig. 1). The culture medium consisted of 50\% Waymouth's medium (GIBCO) and 50\% fetal calf serum (GIBCO) supplemented with 5,000 units/liter penicillin and 5,000 $\mu \mathrm{g} /$ liter streptomycin. A single embryo was placed in a 10 $\mathrm{ml}$ screw-cap plastic test tube containing 1.5 $\mathrm{ml}$ of medium. Each test tube was gassed with

Received January 23, 1980; accepted August 16, 1980 
TABLE 1. Agents tested

\begin{tabular}{|c|c|c|}
\hline Agent & $\operatorname{Dose}^{\mathrm{e}}(\mathrm{mg} / \mathrm{kg})$ & Administration \\
\hline Thiadiazole ${ }^{a}$ in water & 100 & IP \\
\hline Cadmium sulfate in water & 2 & IP \\
\hline Dibrom $^{b}$ in corn oil & 40 & IP \\
\hline Dinoseb $^{c}$ in corn oil & 10 & IP \\
\hline Lead nitrate in water & 50 & IP \\
\hline $\mathrm{PBB}^{\mathrm{d}}$ in sesame oil & 800 & Oral \\
\hline Sodium arsenate in water & 30 & IP \\
\hline Trypan blue in water & 140 & IP \\
\hline
\end{tabular}

a mixture of $10 \% \mathrm{O}_{2}, 5 \% \mathrm{CO}_{2}$, and $85 \% \mathrm{~N}$, and placed on a rotator and rotated at 30-40 rpm in an incubator at $37^{\circ} \mathrm{C}$. At 12,24 , and 36 hours in culture, the $\mathrm{O}_{2}$. concentration was increased to $20 \%, 50 \%$ and $80 \%$, respectively, with $\mathrm{N}$ concomitantly decreased and $\mathrm{CO}_{2}$ maintained constant. Cultivation was terminated at 24 or 42 hours and the development of the embryos evaluated.

After 24 hours in culture, two thirds of the embryos were recovered for examination. The remaining one third was transferred to fresh media and allowed to continue in cultivation until 42 hours. Recovered embryos were examined for rotation of the embryonic axis, heart rate, establishment of the visceral yolk sac circulation, somite number, growth of the limb buds, closure of the neural tube, and development of the allantois and amnion. The results were analyzed by the Student's t-test.

\section{RESULTS}

Because two thirds of the embryos were examined at 24 hours, there were often too few embryos left at 42 hours for a meaningful analysis. We only report, therefore, the statistical analyses of the results at 24-hour cultivation. Days 9 and 10 embryos placed in culture survived well for the duration of the experiment (Table 2), and development compared favorably with development in vivo (Fig. 2).

Table 3 presents the results to be described below. Axial rotation was completed in the majority of the control embryos at 24 hours and only $1 \%$ had failed to rotate at $\mathbf{4 2}$ hours. Maternal treatment with each of the test compounds at either day 9 or 10 of gestation significantly ( $\mathrm{P}=0.05$ or less) retarded rotation of the embryos during the first 24 hours in culture. Continued culture to 42 hours, however, gave variable results, although the trend was for an increase in the number of embryos rotating between 24 and 42 hours. Only dinoseb and trypan blue seemed still to be inhibitory at 42 hours.

At the time of embryo recovery, all embryos had open neural tubes. After 24 hours in culture, $97 \%$ of control embryos had closed neural tubes. Each chemical administered at day 9 of pregnancy induced a significant $(\mathrm{P}=$ 0.05 or less) delay in neural tube closure in the cultured embryos, and at 42 hours there were still many embryos with open neural tubes. Treatment at day 10 was less inhibitory than treatment at day 9; only aminothiadiazole (thiadiazole), 2-(sec-Butyl)-4,6-dinitrophenol (dinoseb), polybrominated biphenyls (PBB), and trypan blue significantly $(\mathrm{P}=0.05$ or less) reduced the incidence of neural tube closure at 24 hours.

There are no anterior limb buds present in embryos at the time of their recovery for culture. Twenty-four hours later, approximately two thirds of the cultured control embryos had anterior limb buds, and at 42 hours almost all controls developed them. Each compound, except dinoseb, administered at day 9 induced a significant ( $\mathrm{P}=0.05$ or less) delay in the appearance of the anterior limb buds. After maternal treatment at day 10 , thiadiazole, 1,2-dibromo-2,2-dichloroethyl dimethyl phosphate (dibrom), lead, arsenic, and trypan blue induced a significant delay at 24 hours. 

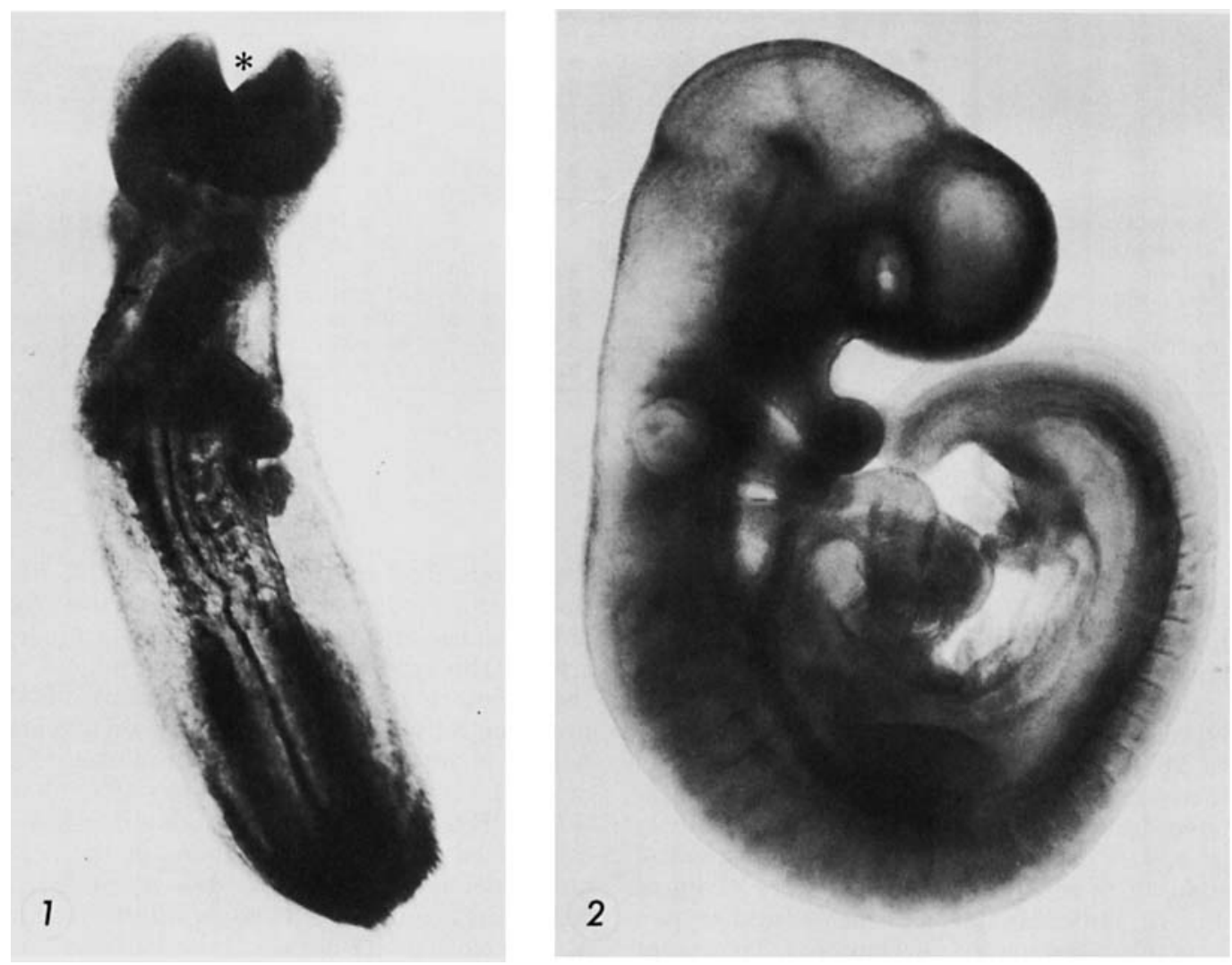

Fig. 1. Rat embryo at time of recovery for culture. Note presence of open neural folds $(*)$.

Fig. 2. Rat embryo after 24 hours in culture.

TABLE 2. Effect of treatment on embryo survival in culture

\begin{tabular}{|c|c|c|c|c|c|c|}
\hline \multirow[b]{2}{*}{ Agent } & \multicolumn{2}{|c|}{$\begin{array}{l}\text { No. of pregnant } \\
\text { rats treated }\end{array}$} & \multicolumn{2}{|c|}{$\begin{array}{l}\text { No. of embryos } \\
\text { placed in culture }\end{array}$} & \multicolumn{2}{|c|}{$\begin{array}{c}\text { Survivors at } 24 \\
\text { hours } \\
(\%)\end{array}$} \\
\hline & Day 9 & Day 10 & Day 9 & Day 10 & Day 9 & Day 10 \\
\hline Thiadiazole & 3 & 11 & 27 & 70 & $93 \pm 5$ & $86 \pm 4$ \\
\hline Cadmium sulfate & 6 & 2 & 55 & 19 & $82 \pm 5$ & $84 \pm 9$ \\
\hline Dibrom & 8 & 2 & 80 & 15 & $87 \pm 8$ & $93 \pm 8$ \\
\hline Dinoseb & 5 & 6 & 38 & 46 & $92 \pm 4$ & $96 \pm 3$ \\
\hline Lead nitrate & 5 & 7 & 44 & 55 & $86 \pm 5$ & $84 \pm 5$ \\
\hline PBB & 7 & 6 & 67 & 31 & $90 \pm 4$ & $97 \pm 3$ \\
\hline Sodium arsenate & 8 & 7 & 80 & 50 & $93 \pm 3$ & $94 \pm 5$ \\
\hline Trypan blue & 6 & 5 & 56 & 43 & $82 \pm 5$ & $86 \pm 5$ \\
\hline Control $^{\mathrm{a}}$ & 41 & 41 & 385 & 271 & & 10 \\
\hline
\end{tabular}

a Pooled control values from all experiments.

- Embryos alive at 24 hours and continued in culture were still alive at 42 hours, when the experiment was terminated. 
TABLE 3. Effect of treatment on embryonic development at 24 hours in culture

\begin{tabular}{|c|c|c|c|c|c|c|c|c|}
\hline \multirow[b]{2}{*}{ Agent } & \multicolumn{2}{|c|}{$\begin{array}{c}\text { Rotated embryonic } \\
\text { axis (\%) }\end{array}$} & \multicolumn{2}{|c|}{$\begin{array}{c}\text { Closed neural tubes } \\
(\%)\end{array}$} & \multicolumn{2}{|c|}{$\underset{(\%)}{\text { Anterior limb buds }}$} & \multicolumn{2}{|c|}{ No. of somites present } \\
\hline & Day 9 & Day 10 & Day 9 & Day 10 & Day 9 & Day 10 & Day 9 & Day 10 \\
\hline Control & $96 \pm 4$ & $95 \pm 5$ & $98 \pm 5$ & $97 \pm 5$ & $61 \pm 17$ & $71 \pm 15$ & $18 \pm 1.0$ & $19 \pm 0.8$ \\
\hline Thiadiazole & $68 \pm 11$ & $61 \pm 11$ & $75 \pm 10$ & $55 \pm 8$ & $5 \pm 5$ & $12 \pm 5$ & $16 \pm 1.0$ & $13 \pm 1.0$ \\
\hline Cadmium sulfate & $81 \pm 6$ & $88 \pm 8$ & $44 \pm 10$ & $88 \pm 12$ & $33 \pm 9$ & $71 \pm 18$ & $16 \pm 0.8$ & $22 \pm 3.0$ \\
\hline Dibrom & $76 \pm 5$ & $86 \pm 10$ & $48 \pm 7$ & 100 & $28 \pm 7$ & $29 \pm 18$ & $16 \pm 0.5$ & $18 \pm 2.0$ \\
\hline Dinoseb & $74 \pm 7$ & $86 \pm 5$ & $75 \pm 8$ & $69 \pm 8$ & $52 \pm 10$ & $73 \pm 9$ & $18 \pm 0.7$ & $20 \pm 0.5$ \\
\hline Lead nitrate & $58 \pm 8$ & $80 \pm 6$ & $28 \pm 10$ & $89 \pm 6$ & $19 \pm 9$ & $41 \pm 10$ & $16 \pm 0.8$ & $18 \pm 1.0$ \\
\hline PBB & $88 \pm 1$ & $79 \pm$ & $70 \pm 8$ & $83 \pm$ & $24 \pm 8$ & $59 \pm 12$ & $16 \pm 0.8$ & $20 \pm 1.0$ \\
\hline Sodium arsenate & $70 \pm 5$ & $83 \pm 6$ & $30 \pm 19$ & $93 \pm$ & $30 \pm 7$ & $48 \pm 10$ & $15 \pm 1.0$ & $17 \pm 1.0$ \\
\hline Trypan blue & $50 \pm 6$ & $68 \pm 8$ & $43 \pm 9$ & $70 \pm 9$ & $4 \pm 4$ & $22 \pm 8$ & $14 \pm 0.5$ & $15 \pm 0.5$ \\
\hline
\end{tabular}

At 42 hours, apparently only thiadiazole and trypan blue continued to have an effect on limb bud growth, regardless of the day of treatment.

During the initial 24 hours in culture, the number of somites in control embryos was 18 to 19 , and at 42 hours their number had increased to 22. Each tested compound administered at day 9 , except dinoseb, significantly ( $P=0.05$ or less) delayed the development of somites. When the compounds were given at day 10 , only thiadiazole, arsenic, and trypan blue inhibited somite development. Treatment at either day 9 or 10 produced variable results at 42 hours. Only thiadiazole, arsenic, and trypan blue appeared still to inhibit the growth of somites.

In addition to the above, observations were also made on heart rate, establishment of the yolk sac circulation, and development of the allantois and amnion. There was no consistent pattern of effects. It is interesting to note that trypan blue significantly inhibited the establishment of the visceral yolk sac circulation. It has been postulated (Beck et al., '67) that interference with the histiotrophic function of the yolk sac is the basis for the teratogenicity of trypan blue.

\section{DISCUSSION}

Following maternal administration at days 9 and 10 of pregnancy, all the tested compounds interfered with in vitro development at 24 hours. All five of these compounds are teratogenic in the rat. There was no correlation, however, between the types of malformations induced in vivo with trypan blue (Gilman et al., '48), lead (McClain and Becker, '75), arsenic (Beaudoin, '74), thiadiazole
(Beaudoin, '73), and PBB (Beaudoin, '77) and the effects produced in in vitro culture. Apparently, the effect seen in vitro is not predictive of the type of malformation induced in the in utero embryo. As an example, PBB produced only cleft palate and diaphragmatic hernia in rat fetuses from mothers treated at days 11 through 13 of pregnancy (Beaudoin, '77). PBB treatment at day 9 induced only one malformed fetus in 90 survivors. In the present experiment, however, day 9 treatment with PBB interfered with axial rotation, neural tube closure, formation of the anterior limb buds, and somite development-a much greater effect on embryonic development than would be predicted from the results of the in vivo experiments. Perhaps similar interferences occur in vivo but either the embryos die or are able to recover and proceed with development to term, albeit smaller than normal.

Maternal treatment with the tested compounds 24 hours prior to culture resulted in the production of more severe developmental disturbances than maternal treatment four hours prior to culture. Only thiadiazole and trypan blue consistently interfered with in vitro development following a four-hour exposure. These observations may reflect the advance in embryonic development during the 20 -hour period or may reflect the rapidity with which these compounds, or their active metabolites, reach the site of action. When administered maternally at day 9 , all tested compounds delayed axial rotation and inhibited neural tube closure at 24 hours in culture, and all compounds, except dinoseb, delayed the growth of the anterior limb buds and somites. Fewer embryos were affected following maternal treatment at day 10. At 42 hours 
in culture, the results became quite variable and with some compounds quite a bit of "catchup" development had apparently taken place. These observations suggest that all the compounds tested interfered with the rate of development in vitro. A similar interference in vivo could be reflected in growth retardation and the appearance of smaller-than-normal day 20 fetuses at autopsy, a common finding in rats with the teratogens used in this study.

Interference with the closure of the neural tube is less apt to be corrected by "catch-up" growth, for if the neural folds do not meet in the proper framework of space and time, then closure fails to take place. This is supported by the observation that at 42 hours of culture, following maternal treatment at day 9 , there were still many embryos $(\sim 30 \%)$ with open neural tubes. Following treatment at day 10, inhibition of neural tube closure was much less pronounced. In the rats used in this study, the neural tube closes on day 10 or early on day 11. Treatment on the morning of day 10 may not allow sufficient time for any but the fastest-acting teratogens to markedly affect neural tube closure (e.g., thiadiazole and trypan blue).

The results of these experiments demonstrate that a combination of maternal treatment with in vitro culture can be used to evaluate the effect of teratogens on embryonic development in the rat. A major advantage with this procedure is that the mother-placenta-embryo unit is intact during exposure and during the time the agent may be presumed to act. The agent, therefore, would be processed by the mother-placenta-embryo unit, which is not possible when the agent is added to the in vitro culture media. Another advantage is that the exposure of the pregnant animal to the test substance can be in the same manner human exposure would be expected to occur (e.g., aerosol, diet, injection). It is not necessary that the agent be miscible with the culture media. This technique should be useful in such studies as the evaluation of the rapidity of placental transfer of a teratogen, the determination of the minimum ex- posure necessary to induce a teratogenic effect, or the analysis of the ability of embryos to recover after a defined period of exposure to a teratogen.

\section{ACKNOWLEDGMENTS}

This work was supported by EPA grant R805125.

\section{LITERATURE CITED}

Beaudoin, A.R. (1973) Teratogenic activity of 2-amino-1, 3,4-thiadiazole hydrochloride in Wistar rats and the protection afforded by nicotinamide. Teratology, 7:65-72.

Beaudoin, A.R. (1974) Teratogenicity of sodium arsenate in rats. Teratology, 10:153-158.

Beaudoin, A.R. (1977) Teratogenicity of polybrominated biphenyls in rats. Environ. Res., 14:81-86.

Beck, F, J.B. Lloyd, and A. Griffiths (1967) Lysosomal enzyme inhibition by trypan blue: A theory of teratogenesis. Science, 157:1880-1182

Gillman, J., C. Gilbert, and T. Gillman (1948) A preliminary report on hydrocephalus, spina bifida and other congenital anomalies in the rat produced by trypan blue. S. Afr. J. Med. Sci., 13:47-90.

Jolly, J., and C. Lieure (1938) Recherches sur la culture des oeufs des mammif "res. Arch. Anat. Microsc. Morphol. Exp., 34:307-374.

Kochhar, D.M. (1975) The use of in vitro procedures in teratology. Teratology, 11:273-288

McClain, R.M., and B.A. Becker (1975) Teratogenicity, fetal toxicity, and placental transfer of lead nitrate in rats. Toxicol. Appl. Pharmacol., 31:72-82.

New, D.A.T. (1971) Methods for the culture of post-implantation embryos of rodents. In: Methods in Mammalian Embryology. J.C. Daniel, Jr., ed. W.H. Freeman \& Co., San Francisco, pp 305-319.

New, D.A.T. (1978) Whole-embryo culture and the study of mammalian embryos during organogenesis. Biol. Rev., 53:81-122.

New, D.A.T., and P. T. Coppola (1977) Development of a placental blood circulation in rat embryos in vitro. $J$. Embryol. Exp. Morphol,, 37:227-235.

New, D.A.T., P.T. Coppola, and D.L. Cockroft (1976) Comparision of growth in vitro and in vivo of post-implantation rat embryos. J. Embryol. Exp. Morphol., 36: 133-144.

New, D.A.T., and K.F. Stein (1946) Cultivation of postimplantation mouse and rat embryos on plasma clots. $\mathrm{J}$. Embryol. Exp. Morphol., 12:101-111.

Nicholas, J.S., and D. Rudnick (1938) Development of rat embryos of egg clinder to headfold stages in plasma cultures. J. Exp. Zool., 78:205-232.

Wilson, J.G. (1978) Survey of in vitro systems: Their potential use in teratogenicity screening. In: Handbook of Teratology. J.G. Wilson and F.C. Fraser, eds. Plenum Press, New York, pp. 135-153. 